Eva-Maria Konrad

\title{
„Was aber feststand, waren ein paar Daten, Fakten" - Dokufiktion als Grenzfall
}

Wie in Rezensionen und Forschungsbeiträgen gleichermaßen betont wird, ist die Lektüre von dokufiktionalen Texten anstrengend, verwirrend, herausfordernd, manchmal sogar überfordernd. Über Ursula Krechels Roman Landgericht, der mir im Folgenden als Beispiel dienen wird und dem auch das titelgebende Zitat entstammt, befindet etwa Friederike Gösweiner in ihrer Rezension, es sei „irritierend [...], nicht zu wissen, wie weit die Fiktion reicht und wo hier tatsächliche Geschichte beginnt. "1 Es wirke, als sei der Autorin

\begin{abstract}
die Möglichkeit eines Romans, einer literarischen, philosophisch-abstrakten Darstellung, $\mathrm{zu}$ wenig [gewesen] und sie wollte stattdessen eine hieb- und stichfeste, konkrete Anklageschrift formulieren, die zwar poetisch klingt und so tut als sei sie Literatur, aber deren Gewicht doch ein tatsächliches, reales ist. Das ist schade, denn die Unentschiedenheit, die sich daraus ergibt, stört. Bei aller noch so aufwendigen Recherchearbeit kann ein Roman doch nie eine juristische Anklage sein, sondern bleibt Fiktion; und für eine überzeugende literarische Darstellung des Schicksals der Hauptfigur braucht es allzu viel konkrete Aktenrealität nicht. ${ }^{2}$
\end{abstract}

Was an dieser Rezension deutlich wird, ist ein Unbehagen, eine Unzufriedenheit, ja sogar eine gewisse Ratlosigkeit gegenüber dem Text - Reaktionen, die sich auf das Wesensmerkmal der dokufiktionalen Darstellung richten: Es ist genau der für dokufiktionale Texte typische „Grenzgang zwischen Fakt und Fiktion“3, an dem sich die Irritation (und Kritik) der Rezensentin entzündet, und damit steht sie nicht allein. ${ }^{4}$

1 Gösweiner, Friederike. „Ein jüdischer Kohlhaas. Ursula Krechels großer Roman ,Landgericht“ über das Schicksal eines jüdischen Exilanten, der nach Deutschland zurückkehrt und vergeblich nach Gerechtigkeit sucht“. In: literaturkritik.de vom 08.10.2012. https://iteraturkritik.de/public/ rezension.php?rez_id=17203 (24.03.2020).

2 Gösweiner: „Ein jüdischer Kohlhaas“.

3 Bidmon, Agnes. „Streng vertraulich! Dokufiktionales Erzählen als Schreibweise des Politischen in der Literatur der Gegenwart anhand von Ilija Trojanows Macht und Widerstand“. In: Politische Literatur. Begriffe, Debatten, Aktualität. Hg. Christine Lubkoll, Manuel Illi, Anna Hampel. Stuttgart: J. B. Metzler 2019, 421-440, hier 423.

4 Auch Ilse Picaper bewertet Landgericht als „zweifellos interessantes, lesenswertes Buch [...], [jedoch] nicht ohne Herausforderung an den Leser“ (Picaper, Ilse. „(Re)Lectures. Sie wollte Zeugin sein. Zu Ursula Krechel, Landgericht. Roman“. In: Germanica 59 (2016), 205-212, hier 
Doch so verbreitet diese durch Dokufiktionen verursachte Verunsicherung auch sein mag, so erstaunlich erscheint sie auf den ersten Blick in Anbetracht der Tatsache, dass eine literarische Verbindung von Fakten und Fiktionen keinesfalls ungewöhnlich ist. Wie auch immer man die dokufiktionalen Collagen genau beschreibt - als eine Mischung aus realen und fiktiven Bezugsobjekten und/oder als eine Mischung aus fiktionalen und faktualen bzw. dokumentarischen Verfahren oder Darstellungsweisen: ${ }^{5}$ All dies ist aus anderen Texten und Genres wohlbekannt. Kaum ein fiktionaler Text kommt ohne realweltliche Referenzen aus, und doch führt dieses Nebeneinander von realen und fiktiven Figuren, Orten und Sachverhalten üblicherweise nicht zu einer Irritation der Leser. ${ }^{6}$ Und dass sich die in faktualen und fiktionalen Texten üblichen Darstellungsweisen nicht nur miteinander verschränken können, sondern mitunter kaum voneinander zu unterscheiden sind, hat schon Hayden White eindrücklich gezeigt. ${ }^{7}$ Die Frage ist also, wo das spezifische Irritationspotenzial dokufiktionaler Texte herrührt.

Aktuelle fiktionalitätstheoretische Überlegungen können darauf eine überzeugende Antwort geben. Auch wenn in der Forschung nach wie vor keine Einigkeit darüber herrscht, wie „Fiktionalität“ genau zu definieren sei, haben sich in den letzten Jahren doch zunehmend sogenannte „institutionelle Theorien“ der

205). Hermann Weber urteilt, in „Krechels Darstellung verbindet sich - für den Leser unkontrollierbar - Recherchiertes und Erfundenes, Roman und Dokumentation“ (Weber, Hermann. „Landgericht. Roman. Von Ursula Krechel“. In: Neue juristische Wochenschrift 66.11 (2013), 762-763, hier 762). Und Sebastian Hammelehle kritisiert, Ursula Krechels „Roman leidet unter seiner Machart. Er will an einen Menschen erinnern, an das Unrecht, das ihm geschehen ist, das Schicksal eines nur vordergründig wieder eingegliederten Emigranten in der Adenauerzeit thematisieren. Doch Krechel bleibt zu nah an ihrem Stoff“ (Hammelehle, Sebastian. „Deutsche-Buchpreisträgerin Ursula Krechel: Die Gerechtwerderin“. In: Spiegel online vom 9.10.2012. https://www.spiegel.de/kultur/literatur/danksagung-der-buchpreistraegerin-ursulakrechel-in-frankfurt-am-main-a-860202.html (24.03.2020)).

5 Vgl. dazu kritisch Christian von Tschilschke, der zu Recht auf die „unvermeidliche[] semantische[] Unschärfe“ des Begriffes „Dokufiktion“ hingewiesen hat: „Denn entweder wird dabei das Dokumentarische mit dem Faktischen gleichgesetzt, wodurch der Bedeutungsunterschied zwischen ,dokumentarisch“ und ,faktisch verwischt wird, oder es werden mit ,Dokument‘ und ,Fiktion' zwei Begriffe einander gegenübergestellt, die nicht auf derselben logischen Anschauungsebene liegen“ (Tschilschke, Christian von. „Biographische Dokufiktion in der spanischen Literatur der Gegenwart. Las esquines del aire von Juan Manuel de Prada und Soldados de Salamina von Javier Cercas“. In: Literatur als Lebensgeschichte. Biographisches Erzählen von der Moderne bis zur Gegenwart. Hg. Peter Braun, Bernd Stiegler. Bielefeld: transcript 2012, 377-400, hier 381).

6 Ich verwende durchgehend das generische Maskulinum, will damit aber ausdrücklich alle Geschlechter eingeschlossen wissen.

7 Vgl. White, Hayden. Metahistory. The Historical Imagination in Nineteenth-Century Europe. Baltimore/London: Johns Hopkins University Press 1973. 
Fiktionalität durchgesetzt, die den „Begriff der Fiktionalität über die regelgeleiteten Handlungen und Einstellungen von Personen (und nicht beispielsweise über die semantischen Eigenschaften von Sätzen oder die ontologischen Eigenschaften von Referenzobjekten) bestimm[en]"8. Fiktionalität wird also begriffen als ein durch (synchron und diachron variable) Konventionen festgelegter Umgang mit bestimmten Werken, zu dem sich Autoren und Leser gleichermaßen verpflichtet fühlen.

Einer der Vorteile einer institutionellen Fiktionalitätstheorie für die Analyse von Dokufiktionen liegt damit sofort auf der Hand: Die institutionelle Grundidee einer regelgeleiteten Fiktionalitätspraxis ist nicht an fiktionale literarische Texte gebunden, sondern lässt sich auch auf andere Medien (wie z. B. den Film) übertragen. ${ }^{9}$ Gerade für ein intermedial so verbreitetes Phänomen wie die Dokufiktion ist dieser Ansatz deshalb überaus attraktiv. Auch wenn die konkreten Konventionen der aktuellen Fiktionalitätspraxis im Folgenden am Beispiel literarischer Texte nachgezeichnet werden, sollten die Überlegungen und Rückschlüsse also durch kleinere Veränderungen in den Formulierungen auf andere dokufiktionale Werke übertragbar sein.

\section{1.}

Die aktuelle Fiktionalitätspraxis regelt den Umgang, den Autoren wie Leser mit bestimmten Werken pflegen. In Bezug auf die Autorseite bedeutet dies konkret, dass ein Text nur dann ein fiktionaler ist, wenn er mit einer „Fiktionalitätsintention“, d. h. „mit der Absicht hervorgebracht wurde, gemäß den Konventionen der Fiktionalitätsinstitution rezipiert zu werden." 10 Der Autor muss also beabsichtigt haben, dass die Leser den Text und die in ihm enthaltenen Äußerungen auf ge-

8 Köppe, Tilmann. „Die Institution Fiktionalität“. In: Fiktionalität. Ein interdisziplinäres Handbuch. Hg. Tobias Klauk, Tilmann Köppe. Berlin/Boston: De Gruyter 2014, 35-49, hier 41. Der Grundstein der institutionellen Fiktionalitätstheorie wurde von Peter Lamarque und Stein Haugom Olsen in ihrem wegweisenden Buch Truth, Fiction, and Literature gelegt. Vgl. Lamarque, Peter/Olsen, Stein Haugom. Truth, Fiction, and Literature. A Philosophical Perspective. Oxford: Oxford University Press 2002 [1994].

9 Vgl. auch schon Köppe: „Die Institution Fiktionalität“, 47: „Die institutionelle Theorie der Fiktionalität empfiehlt sich als Grundlage einer medienübergreifenden Theorie“.

10 Köppe: „Die Institution Fiktionalität“, 35. Bezug nehmend auf die maßgebliche institutionelle Theorie von Lamarque und Olsen erläutert Köppe weiter: „Fiktionale literarische Werke haben demnach ihren Ursprung in fiktionalen Äußerungsakten. Für diese Akte ist konstitutiv, dass ein Sprecher natürlichsprachliche Sätze in der Absicht äußert, dass Leser den Sätzen gegenüber 
nau die Weise rezipieren, die die aktuelle Fiktionalitätspraxis für sie vorsieht. Um diese Intention für die Leser erkennbar zu machen, ist der Autor des Weiteren verpflichtet, den Text mit ebenfalls durch Konventionen festgelegten Fiktionalitätssignalen auszuzeichnen - z. B. durch eine entsprechende Markierung im Paratext oder durch spezielle „textinterne Merkmale [...], die eine übermenschliche Allwissenheit des Erzählers voraussetzen; dazu gehören insbesondere Einblicke des Erzählers in die Gedanken- und Gefühlswelt seiner Figuren. "11

Über die Normen, die die fiktionalitätstypische Rezeptionshaltung bestimmen, herrscht in der Forschung keine vollständige Einigkeit, im Wesentlichen scheinen sie aber in den folgenden Ge- und Verboten zu bestehen: „Verboten ist es den Adressaten fiktionaler Äußerungen, vom Gehalt der Äußerungen (unmittelbar) auf das Bestehen der beschriebenen Sachverhalte in der Wirklichkeit zu schließen."12 Ebenfalls verboten ist den Lesern ein Rückschluss von den fiktionalen Äußerungen auf eine entsprechende Überzeugung des Autors, d. h. die fiktionalen Äußerungen erlauben kein Urteil darüber, ob der Autor die beschriebenen Sachverhalte für wahr hält oder nicht: „In fiktionalen Äußerungen, das zeigt das Verbot von Schlüssen auf Überzeugungen sowie weitere Einstellungen des Sprechers, sind die in nicht-fiktionalen Kontexten gültige[n] Bedingungen für Sprechakte (zumindest teilweise) aufgehoben. "13 Zu diesen Verboten für die Rezipienten gesellt sich das Gebot, in eine „imaginative Auseinandersetzung mit [den] fiktionalen Äußerungen“"14 zu treten. Dieses Gebot betrifft zwei unterschiedliche Aspekte: Zum einen sind die Leser dazu aufgefordert, sich vorzustellen, dass die fiktionalen Äußerungen des Autors reale bzw. authentische Äußerungen eines (vom Autor üblicherweise verschiedenen) Erzählers sind. ${ }^{15}$ Zum anderen sollen die Re-

eine fiktionstypische Rezeptionshaltung (,fictive stance) einnehmen.“ (Köppe: „Die Institution Fiktionalität“, 35-36).

11 Klein, Christian/Martínez, Matías. „Wirklichkeitserzählungen. Felder, Formen und Funktionen nicht-literarischen Erzählens“. In: Wirklichkeitserzählungen. Felder, Formen und Funktionen nicht-literarischen Erzählens. Hg. Christian Klein, Matías Martínez. Stuttgart/Weimar: J. B. Metzler 2009, 1-13, hier 3.

12 Köppe: „Die Institution Fiktionalität“, 36.

13 Köppe: „Die Institution Fiktionalität“, 37.

14 Köppe: „Die Institution Fiktionalität“, 37.

15 Vgl. Köppe: „Die Institution Fiktionalität“, 37. Vgl. dazu auch Matías Martínez und Michael Scheffel: „Der Autor produziert also Sätze, die zwar real, aber inauthentisch sind - denn sie sind nicht als Behauptungen des Autors zu verstehen. Dem fiktiven Erzähler hingegen sind dieselben Sätze als authentische Sätze zuzuschreiben, die aber imaginär sind - denn sie werden vom Erzähler behauptet, jedoch nur im Rahmen einer imaginären Kommunikationssituation “ (Martínez, Matías/Scheffel, Michael. Einführung in die Erzähltheorie. München: C.H.Beck 2002 [1999], 17). Gertken und Köppe weisen zu Recht darauf hin, dass das formulierte Gebot eigentlich um den 
zipienten „die Sätze des Werkes zum Anlass nehmen [...], sich relevante Konturen einer Welt vorzustellen"16 - eine vorsichtige Formulierung, die einerseits zum Ausdruck bringt, dass Leser das im Text Dargestellte imaginieren sollen, die andererseits aber auch berücksichtigt, dass es selbstverständlich kein „einfaches Entsprechungsverhältnis zwischen fiktionalen Sätzen und vorzustellenden Sachverhalten gibt"17: Weder dürfen sich die Leser nur das vorstellen, was im Text gesagt wird - sie müssen darüber hinaus Leerstellen imaginativ füllen, die der Text offenlässt -, noch können sich die Leser an jeder Stelle genau das vorstellen, was im Text gesagt wird - gerade bildhafte rhetorische Mittel lassen häufig einen großen imaginativen Freiraum. Zusammengefasst bestehen die Konventionen der aktuellen Fiktionalitätspraxis also zum einen in der „Fiktionalitätsintention“ des Autors, die durch entsprechende Fiktionalitätssignale angemessen zum Ausdruck gebracht werden muss, und zum anderen auf der Rezipientenseite sowohl in dem Verbot, auf die Wahrheit des Gesagten und auf die Überzeugung des Autors von der Wahrheit des Gesagten zu schließen, als auch in dem Gebot, sich das Gesagte und einen Erzähler, der das Gesagte äußert, vorzustellen. ${ }^{18}$

\section{2.}

Vor diesem Hintergrund wird nun schnell klar, warum Dokufiktionen für Irritation sorgen müssen: Unklar ist erstens, ob dokufiktionale Werke von ihren Autoren mit einer Fiktionalitätsintention hervorgebracht wurden, d. h. ob sie mit der Absicht verfasst wurden, dass die Rezipienten ihnen gegenüber die für fiktionale Werke typische Haltung einnehmen sollen. Entsprechend häufig zeigen auch die Signale, die den Text als fiktional oder faktual ausweisen sollen, in unterschiedliche Richtungen: Ursula Krechels Landgericht etwa wird im Paratext einerseits als „Roman“ bezeichnet, beinhaltet aber ebenso „Nachweise“19 über diverse Ar-

Zusatz erweitert werden müsste, dass „Leser sich [nur] insoweit eine konkrete Erzählinstanz als Sprecher vorstellen und gedanklich ausmalen sollen, als dies aufgrund der Textgrundlage sinnvoll ist (wobei dies die Möglichkeit umfasst, dass es nicht sinnvoll ist).“ (Gertken, Jan/Köppe, Tilmann. „Fiktionalität“. In: Grenzen der Literatur. Zu Begriff und Phänomen des Literarischen. Hg. Simone Winko, Fotis Jannidis, Gerhard Lauer. Berlin/New York: De Gruyter 2009, 228-266, hier 261).

16 Köppe: „Die Institution Fiktionalität“, 38.

17 Köppe: „Die Institution Fiktionalität“, 38.

18 Zur Diskussion weiterer Ge- und Verbote vgl. Köppe: „Die Institution Fiktionalität“, 38-43, und Lamarque/Olsen: Truth, Fiction, and Literature, Kap. 2.

19 Krechel, Ursula. Landgericht. München: btb 2014 [2012], 511. 
chive und Quellen, aus denen die Autorin geschöpft hat und die sie im Text zum Teil wörtlich zitiert. Fragwürdig erscheint bei Dokufiktionen deshalb des Weiteren, ob die Rezipienten wirklich nicht auf die Wahrheit des Dargestellten oder auf die Überzeugung der Autoren von der Wahrheit des Gesagten schließen dürfen. Es ist nicht einmal klar, ob die Rezipienten tatsächlich primär dazu angehalten sind, sich das Gesagte (und einen entsprechenden Sprecher) vorzustellen - oder ob sie die Ausführungen nicht vielmehr mit ihren bisherigen Überzeugungen abgleichen oder Erkenntnisse daraus gewinnen sollen. Auch wenn die aktuelle Fiktionalitätspraxis möglicherweise auf Teile der dokufiktionalen Werke anwendbar ist (darauf wird später zurückzukommen sein), zeigt sich doch sehr klar, dass Dokufiktionen als Gesamttexte den Konventionen für die Produktion wie für die Rezeption fiktionaler Texte nicht (oder zumindest nicht eindeutig) entsprechen.

Offenkundig gelten aber genauso wenig die üblichen Regeln der faktualen Kommunikation. Indem Autoren Texte ,als faktual markieren, sichern sie zu, dass sie wahrhaftig, knapp, klar und relevant berichten. “20 Die Autoren faktualer Texte verpflichten sich auf die Wahrheit, auf den Informationsgehalt, auf eine mögliche Rechtfertigung sowie auf die Überzeugung von der Wahrheit des Gesagten ${ }^{21}$ und dürfen im Gegenzug erwarten, dass die Leser den Text entsprechend rezipieren. Auch dies trifft möglicherweise auf Teile dokufiktionaler Werke zu, auf den Gesamttext aber sicherlich nicht: Der Leser darf weder davon ausgehen, dass alles Dargestellte der Wahrheit entspricht, noch darf er auf eine entsprechende Überzeugung des Autors schließen etc. Die durch Dokufiktionen ausgelöste Irritation lässt sich deshalb am besten so beschreiben, dass der Leser in ständigem Zweifel darüber ist, welchen Konventionen er bei der Lektüre des Textes nun folgen darf oder sogar muss, da Dokufiktionen offenbar sowohl mit den Regeln des faktualen als auch mit denjenigen des fiktionalen Diskurses brechen.

\section{3.}

Wie ist nun mit diesem Befund umzugehen? Aus fiktionalitätstheoretischer Perspektive sind drei Möglichkeiten denkbar: Erstens ließe sich angesichts der Mischung aus Fakt und Fiktion, wie sie in Dokufiktionen auftritt, für eine Gra-

20 Klein/Martínez: „Wirklichkeitserzählungen“, 3.

21 Vgl. zu diesen semantischen und pragmatischen Regeln für faktuale Behauptungen Searle, John R. „The Logical Status of Fictional Discourse“ [1974/75]. In: Expression and Meaning. Studies in the Theory of Speech Acts. Hg. John R. Searle. Cambridge: Cambridge University Press 1979, 58-75, hier 62. 
duierung der Fiktionalität (bzw. Faktualität) argumentieren. Zweitens scheinen Dokufiktionen mithilfe einer kompositionalistischen Analyse greifbar zu werden, d. h. mit einem theoretischen Zugang, dem zufolge fiktionale Texte nicht vollständig aus fiktionalem Diskurs bestehen müssen, sondern auch faktualen Diskurs integrieren können. Die dritte Möglichkeit, Dokufiktionen theoretisch einzuhegen, besteht schließlich darin, Dokufiktionen als echte fiktionalitätstheoretische „Grenzfälle“ zu begreifen, d. h. als Werke, bei denen unklar ist, „ob ein Text mit der fraglichen kategorialen Absicht [d.h. der Fiktionalitätsintention] hervorgebracht wurde." 22

\section{1}

Die erste Option, die Annahme einer Graduierung der Fiktionalität, erscheint zunächst insofern als attraktiv, als das konkrete „Mischverhältnis“ zwischen Dokumentarischem und Fiktionalem nicht nur von Text zu Text, ${ }^{23}$ sondern auch innerhalb eines dokufiktionalen Textes stark variieren kann: In Ursula Krechels Landgericht etwa finden sich auf den ersten ca. 400 Seiten nur recht verstreut dokumentarische Einfügungen, während die letzten 100 Seiten übervoll von ihnen sind. Sind die letzten Kapitel von Landgericht damit als weniger fiktional zu bewerten als der Rest des Buches? Oder auf Dokufiktion im Allgemeinen gewendet: Sind dokufiktionale Werke als weniger fiktional bzw. als faktualer zu beurteilen als „normale“ Romane?

Vor dem Hintergrund der vorgestellten institutionellen Fiktionalitätstheorie scheidet diese Möglichkeit trotz einer gewissen Anfangsplausibilität aus: Zum einen lässt sich nicht sinnvoll erklären, wie die Autoren dokufiktionaler Texte ihre Werke mehr oder weniger mit der Intention hervorgebracht haben könnten, dass die Rezipienten diesen Texten gegenüber die fiktionalitätstypische Rezeptionshaltung einnehmen mögen. Fiktionalitätsintentionen gehorchen (wie Intentionen allgemein) „einer binären Logik“24, d. h. Autoren haben entweder eine Fiktionalitätsintention oder sie haben sie nicht. Zum anderen lässt sich aber auch nicht verständlich machen, inwiefern die Rezipienten dokufiktionaler Texte mehr oder weniger nicht auf die Wahrheit des Gesagten schließen dürfen oder sich den Inhalt der Texte mehr oder weniger vorstellen sollen etc. Zudem ist offenkundig,

22 Köppe, Tilmann. Literatur und Erkenntnis. Studien zur kognitiven Signifikanz fiktionaler literarischer Werke. Paderborn: mentis 2008, 45.

23 Vgl. Oels, David/Porombka, Stephan/Schütz, Erhard. „DokuFiktion - Editorial“. In: Non Fiktion. Arsenal der anderen Gattungen: Dokufiktion 1/2 (2006), 108-111.

24 Köppe: Literatur und Erkenntnis, 44. 
dass eine Erwägung des numerischen Verhältnisses von fiktiven und faktischen Elementen oder von fiktionalen und faktualen Passagen in unserer aktuellen Fiktionalitätspraxis keine Rolle spielt. Fiktionalität und Faktualität bemessen sich nicht quantitativ nach der Anzahl bestimmter Elemente, sondern markieren einen qualitativen Unterschied in der Produktion und Rezeption von Texten. Eine graduelle Interpretation der aktuellen Fiktionalitätskonventionen ist also nicht sinnvoll möglich, ${ }^{25}$ sodass sich die Annahme eines graduellen Fiktionalitätsbegriffs (nicht nur) für die Analyse von Dokufiktion ausschließt.

\section{2}

Eine zweite Möglichkeit, Dokufiktion theoretisch einzufangen, besteht in einem kompositionalistischen Fiktionalitätsverständnis. Markus Wiegandt etwa scheint „mit der kompositionalistischen Explikation von Fiktion eine brauchbare fiktionstheoretische Grundlage gefunden, um sich mit Phänomenen der ,Dokufiktion` auseinander zu setzen [sic]." 26 Anders als Peter Blume, auf dessen terminologisch unscharfe Überlegungen sich Wiegandt dabei stützt, ${ }^{27}$ begreife ich den Kompositionalismus - wie bereits angedeutet - als diejenige fiktionalitätstheoretische

25 Vgl. dazu schon Gertken/Köppe: „Fiktionalität“, 260, Fn. 84: „,[E]s ist unklar, woher die Gradualität des Begriffs eigentlich kommen (bzw. worauf sie beruhen) soll. Erstens ist es sicher nicht so, dass die für fiktionale Texte konstitutive Intention mehr oder weniger stark vorliegt. (Sollte das heißen, dass man die Fiktionalität eines Textes öfters nur halbherzig intendiert?) Zweitens scheint nicht der Fall zu sein, dass die Gradualität auf dem Inhalt der Intention beruht (dies würde besagen, dass man einen Text mehr oder weniger als prop gebrauchen soll)." Vgl. an anderer Stelle auch noch einmal Köppe, der zu Recht darauf hinweist, dass bei der Diskussion um Grade der Fiktionalität häufig „eigentlich die Genese, die Interpretation oder die epistemischen Funktionen der Texte, nicht ihre Fiktionalität im engeren Sinne“ (Köppe: „Die Institution Fiktionalität“, 47) zur Debatte stehen. Vgl. zu einer ausführlicheren Diskussion von Graden der Fiktionalität auch Konrad, Eva-Maria. Dimensionen der Fiktionalität. Analyse eines Grundbegriffs der Literaturwissenschaft. Münster: mentis 2014, Kap. 4.2.2.1.

26 Wiegandt, Markus. Chronisten der Zwischenwelten. Dokufiktion als Genre. Operationalisierung eines medienwissenschaftlichen Begriffs für die Literaturwissenschaft. Heidelberg: Winter 2017, 39. 27 Problematisch an Blume ist unter anderem, dass sich seine Fiktionalitätsdefinition nur auf Gesamttexte, nicht aber auf die für Kompositionalisten eigentlich relevante lokale Ebene einzelner Propositionen bzw. Textpassagen anwenden lässt (vgl. Blume, Peter. Fiktion und Weltwissen. Der Beitrag nichtfiktionaler Konzepte zur Sinnkonstitution fiktionaler Erzählliteratur. Berlin: Erich Schmidt 2004, 78). Darüber hinaus wird durch seine unscharfe Sprechweise von einer kompositionalistischen Mischung „aus fiktionalen und nichtfiktionalen Elementen“ (Blume: Fiktion und Weltwissen, 23) letztlich nie völlig klar, ob es sich seiner Meinung nach nun um eine Mischung auf der Objekt- oder auf der Darstellungsebene handelt. Vgl. zu einer ausführlichen Kritik an Blume Konrad: Dimensionen der Fiktionalität, Kap. 4.1.1.1. 
Position, die fiktionale Texte als eine potenzielle Mischung aus fiktionalem und faktualem Diskurs bzw. aus fiktionalen und faktualen Textpassagen versteht. Der Kompositionalismus steht damit in Konkurrenz zum Autonomismus, dem zufolge fiktionale Texte ausschließlich aus fiktionalem Diskurs bestehen, und zum Panfiktionalismus, der alle Texte unterschiedslos als fiktional beurteilt. Während der berühmte erste Satz aus Tolstois Anna Karenina - „Alle glücklichen Familien sind einander ähnlich, jede unglückliche Familie ist unglücklich auf ihre Weise.“28 also sowohl von Autonomisten als auch von Panfiktionalisten als fiktional bewertet wird, besteht für Kompositionalisten grundsätzlich die Möglichkeit, diese Äußerung (auch) als faktualen Diskurs zu interpretieren.

So verstanden scheint der Kompositionalismus nun tatsächlich für eine Analyse dokufiktionaler Texte von Vorteil zu sein: Erstens scheint die Strategie gewinnbringend, die für die Beurteilung des dokufiktionalen Gesamttextes problematische Vermengung von Fiktionalem und Dokumentarischem ,aufzuschlüsseln“ in eine Analyse einzelner Textpassagen und damit die globale Bewertung durch eine lokale zu ersetzen. Zweitens bieten sich gerade dokufiktionale Texte für eine derartige lokale Analyse an, weil sie es dem Leser - anders als „normale“ fiktionale Texte - in den meisten Fällen ersparen, erst mühsam nach Faktualitätssignalen suchen zu müssen, d. h. nach Hinweisen darauf, dass eine konkrete Passage in einem fiktionalen Text (auch) als faktualer Diskurs zu interpretieren ist: In Ursula Krechels Landgericht etwa finden sich die dokumentarischen Passagen in Kursivschrift. ${ }^{29}$ Dadurch, dass das Dokumentarische also schon rein optisch vom übrigen Diskurs abgehoben ist, hat der Leser keine Probleme mit dessen Identifikation. Drittens scheint der Kompositionalismus aber auch auf

Wiegandt selbst konstatiert über seine kompositionalistische Sichtweise mit Bezug auf Blume: „In diesem Sinne verstehe auch ich Fiktionalität als Texteigenschaft literarischer Texte, die fiktive Elemente integrativ neben Elemente mit außertextuellem Referenzrahmen setzt“ (Wiegandt: Chronisten der Zwischenwelten, 39). Von meiner eigenen Position weicht dies nicht nur deshalb deutlich ab, weil der Kompositionalismus hier klar über die Referenzobjekte definiert wird - eine Festlegung, die in dieser Klarheit bei Blume nicht zu finden ist und der auch mein pragmatischer Ansatz entgegensteht. Darüber hinaus steht die Auffassung von ,Fiktionalität als Texteigenschaft literarischer Texte“ (Wiegandt: Chronisten der Zwischenwelten, 39) einer institutionellen Fiktionalitätstheorie entgegen und scheint eher ein textimmanentes bzw. produktorientiertes Fiktionalitätsverständnis nahezulegen. Zu den Problemen produktorientierter Theorien, die Fiktionalität ohne Bezugnahme auf die Intentionen des Autors und das Verhalten des Rezipienten definieren, vgl. Konrad: Dimensionen der Fiktionalität, Kap. 4.1.2.2.1.

28 Tolstoi, Lew. Anna Karenina. Aus dem Russischen neu übersetzt und kommentiert von Rosemarie Tietze. München: Carl Hanser 2013 [1878], 7.

29 Um nur noch ein weiteres Beispiel zu nennen: Auch in Ilija Trojanows Macht und Widerstand sind die integrierten Auszüge aus realen Dokumenten graphisch abgesetzt. Vgl. dazu den informativen Aufsatz von Bidmon: „Streng vertraulich!“, hier v. a. 437. 
das für Dokufiktion typische und wiederholt konstatierte Ineinandergreifen des Dokumentarischen und Fiktionalen angemessen reagieren zu können. Christian von Tschilschke etwa definiert „Dokufiktion“ dezidiert als „einen medien- und gattungsübergreifenden Darstellungsmodus, in dem sich dokumentarische und fiktionale Elemente, Verfahren und Strategien verschränken“30. Der Kompositionalismus scheint diese Verschränkung theoretisch reflektieren zu können, da er nicht von einem Wechsel von fiktionalen und faktualen Passagen ausgehen muss, sondern - zumindest im Rahmen eines pragmatischen, sprechaktbasierten Ansatzes - das gleichzeitige Vorhandensein eines fiktionalen und faktualen Sprechaktes annehmen kann. ${ }^{31}$ Dies gilt für kompositionalistische Paradebeispiele wie den ersten Satz aus Anna Karenina, der ja klarerweise nicht losgelöst vom weiteren Inhalt des Romans ist, sondern diesen schon thematisch vorwegnimmt. Doch auch die dokumentarischen Einschübe in Dokufiktionen scheinen geeignete Kandidaten für eine derartige Analyse zu sein. So erzählt Ursula Krechel in Landgericht die Geschichte Richard Kornitzers (realiter: Robert Bernd Michaelis), einem deutschen Richter mit jüdischen Wurzeln, der im Dritten Reich zur Flucht gezwungen ist und nach seiner Rückkehr nach Deutschland Wiedergutmachung und späte Gerechtigkeit sucht. Über diesen Richard Kornitzer heißt es an einer Stelle:

Kornitzer dachte noch einmal an den Apfel, den ihm die junge Frau Dreis wie im Paradies vom Dach des Kleiderschranks geholt hatte, und er fühlte sich unendlich privilegiert, so sehr, daß er keine Worte dafür hatte. [...] Er freute sich an seinem Richteramt, auch der Umgang mit den Beisitzern fiel ihm nicht schwer, und - auf ganz existenzielle Weise - freute er sich zu wohnen, wenigstens zeitweise, wenigstens versuchsweise. Er hatte den kleinen Kindern in Berlin einige Male das Märchen von den Bremer Stadtmusikanten vorgelesen. Etwas Besseres als den Tod findest du überall, hatte er betont. [...] Etwas Besseres als den

30 von Tschilschke: „Biographische Dokufiktion in der spanischen Literatur der Gegenwart“, 381-382.

31 Zu einer ausführlichen Begründung dieser Gleichzeitigkeit vgl. Konrad: Dimensionen der Fiktionalität, Kap. 4.2.2.2. Wichtig ist, dass der Kompositionalismus damit jedoch keine Graduierung der Fiktionalität impliziert. Selbst in dem Fall, in dem eine Proposition als fiktional und faktual analysiert wird, liegen beide Intentionen (die Fiktionalitäts- und die Faktualitätsintention) vollumfänglich vor. Der Kompositionalismus gesteht damit also lediglich einem global als fiktional intendierten Diskurs faktuale Ausnahmen zu. Für den Fiktionalitätsbegriff müssen (ebenso wie für den Faktualitätsbegriff) aber auf der globalen Ebene des Gesamttexts und auf der lokalen Ebene einzelner Propositionen dieselben Bedingungen gelten, d. h. wenn man auf der lokalen Ebene einen klassifikatorischen Fiktionalitätsbegriff vertritt - die entsprechende Proposition ist vollumfänglich fiktional oder vollumfänglich faktual -, lässt sich auf der globalen Ebene nicht ohne Selbstwiderspruch ein komparativer Begriff vertreten, der den Gesamttext als mehr oder weniger fiktional (oder faktual) ausweisen würde. 
Tod findest du überall, und er war ein Zeuge dieses richtigen und im richtigen Augenblick zu zitierenden Satzes.

An den Oberbürgermeister der Stadt, an die ihm zugeordnete Betreuungsstelle „Opfer des Faschismus“ hatte er noch vom Dorf über dem See geschrieben: Für Ihre frdl. Anfrage vom 13. ds Mts betr. meine Wohnung in Mainz danke ich Ihnen verbindlichst. Ich habe bisher noch keine Nachricht über den Zeitpunkt meines Dienstantrittes erhalten. Mit Rücksicht auf die Schwierigkeiten der Wohnungsbeschaffung schlage ich vor, daß ich zunächst für kurze Zeit allein dorthin komme. Dafür würde ich nur ein möbliertes Zimmer benötigen. Genau so war es gekommen. Die freundliche Hoffnung auf eine komfortable Zweizimmerwohnung, auf eine Intimität zerschlug sich vor seinen Augen [...].32

Es wäre in der Tat unplausibel, an dieser und zahlreichen vergleichbaren Stellen von einem steten Oszillieren zwischen fiktionalem und faktualem Diskurs auszugehen: Die dokumentarische Passage ist nicht getrennt von der Fiktion, sondern ergänzt und bereichert sie. Dieses Ineinandergreifen scheint der Kompositionalismus angemessen berücksichtigen zu können, indem er die kursivierten Passagen als fiktional und als faktual begreift. Zuletzt kann der Kompositionalismus dadurch auch dem wiederholt betonten ,besonderen Authentizitätsgehalt“"33 von

32 Krechel: Landgericht, 107-108.

33 Bidmon: „Streng vertraulich!“, 429. Vgl. dazu auch den aufschlussreichen Aufsatz von Huck, der sechs Strategien identifiziert, mit denen sich Dokufilme von den Massenmedien abgrenzen und auf diese Weise durch einen „falschen Umkehrschluss“ Glaubwürdigkeit und Authentizität zu gewinnen suchen (vgl. Huck, Christian. „Authentizität im Dokumentarfilm. Das Prinzip des falschen Umkehrschlusses als Erzählstrategie zur Beglaubigung massenmedialen Wissens“. In: Authentisches Erzählen. Produktion, Narration, Rezeption. Hg. Antonius Weixler. Berlin/Boston: De Gruyter 2012, 239-264, hier 252-260). Interessanterweise lassen sich einige der von ihm genannten Aspekte auch auf Krechels Landgericht übertragen: Parallel dazu, dass der Dokumentarfilm laut Huck versucht, die „gezeigte[n] Personen als unverstellt aufzuzeigen“ (Huck: „Authentizität im Dokumentarfilm“, 255), wird auch Richard Kornitzer von Ursula Krechel keineswegs als perfekte Figur dargestellt, sondern erscheint ganz im Gegenteil wiederholt als Unsympath (vgl. Platthaus, Andreas: „Ursula Krechels ,Landgericht‘. In der Sache Kornitzer“. In: Frankfurter Allgemeine Zeitung vom 3.10.2012. https://www.faz.net/aktuell/feuilleton/buecher/rezensionen/belletristik/ursula-krechelslandgericht-in-der-sache-kornitzer-11912092.html?printPagedArticle=true\#pageIndex_2 (07.10.2020), sowie Möller, Barbara: „Der verzweifelte Kampf des Richard Kornitzer“. In: Die Welt vom 30.01.2017. https://www.welt.de/kultur/medien/article161634391/Der-verzweifelteKampf-des-Richard-Kornitzer.html (07.10.2020)). Auch die Autorin Krechel „mischt sich [also ganz wie] der Dokumentarfilmer (vermeintlich) nicht ein ins Leben“ (Huck: „Authentizität im Dokumentarfilm“, 259). In Bezug auf diese Art der Darstellung scheint damit beide Male ein ganz ähnlicher Authentizitätseffekt vorzuliegen, allerdings im Fall des literarischen dokufiktionalen Textes nicht notwendig unter Zuhilfenahme des von Huck für Dokumentarfilme behaupteten falschen Umkehrschlusses. Der wiederholte Wechsel der intradiegetischen Perspektive sowie die teilweise extremen Zeitsprünge durch die großen Analepsen in Landgericht entsprechen 
Dokufiktion und der ihr zugeschriebenen wichtigen Rolle bei der Wissensvermittlung Rechnung tragen: ${ }^{34}$ Lässt man zu, dass fiktionale Texte auch faktualen Diskurs beinhalten können, lässt sich beides erklären.

Als grundsätzliche Anhängerin des Kompositionalismus halte ich diese Theorie auch im Falle der Dokufiktion für attraktiv, weil sie diesem Phänomen wesentlich besser gerecht wird als ihre unmittelbaren Konkurrenten. Der Grund dafür liegt - wie bereits erläutert - vor allem darin, dass der Kompositionalismus auf der lokalen Ebene der Propositionen bzw. einzelner Textpassagen zweifellos eine Reihe von Schwierigkeiten umgehen kann, vor denen der Autonomismus und Panfiktionalismus wohl kapitulieren müssen. Und dennoch bin ich der Überzeugung, dass eine kompositionalistische Analyse letztlich nicht ausreicht, um Dokufiktionen angemessen zu beurteilen. Insbesondere der erste und dritte genannte Vorteil des Kompositionalismus entpuppen sich bei genauerer Betrachtung als nur vordergründige Lösungen der Probleme, die Dokufiktionen bereiten: Weder erübrigt die feingliedrige lokale Analyse einzelner Textpassagen, wie sie der Kompositionalismus möglich macht, ein Urteil über den Status des Gesamttextes, noch kann der Kompositionalimus das für Dokufiktionen typische Ineinandergreifen von Fiktionalem und Dokumentarischem für jede einzelne Textpassage schlüssig erklären.

Um die Schwierigkeiten dieser beiden Aspekte zu verdeutlichen, muss zunächst noch einmal betont werden, dass der Kompositionalismus eine Fiktionalitätstheorie ist - und zwar diejenige Fiktionalitätstheorie, der zufolge fiktionale Texte nicht vollständig aus fiktionalem Diskurs bestehen müssen. Hielte man den Kompositionalimus für den korrekten theoretischen Ansatz zur Analyse von Dokufiktionen, ginge man also gleichzeitig implizit von der These aus, dass dokufiktionale Texte insgesamt, als Gesamttexte, fiktional sind. Damit müssten für die Produktion und Rezeption dokufiktionaler Texte dann aber auch die Regeln der aktuellen Fiktionalitätspraxis gelten. Behauptet wäre also, dass der Text mit einer Fiktionalitätsintention verfasst wurde und dass die Leser ihn deshalb auch mit Ausnahme der als dokumentarische Einfügungen gekennzeichneten Passa-

darüber hinaus der von Huck genannten Strategie des Dokumentarfilms, der „Gradlinigkeit der massenmedialen Erzählung“ (Huck: „Authentizität im Dokumentarfilm“, 258) zuwiderzulaufen. 34 Vgl. etwa Bidmon, die davon spricht, dass „ein wesentliches Element des dokufiktionalen Erzählens die Wissensvermittlung“ sei (Bidmon: „Streng vertraulich!“, 431). Vgl. in Bezug auf die Dokufiktionen in mancherlei Hinsicht nicht unähnlichen „Wissenschaftsthriller“ von Michael Crichton und Frank Schätzing auch Hahnemann, Andy. ,,Footnotes are real‘. Populäre Literatur als Medium der Wissensvermittlung “. In: Non Fiktion. Arsenal der anderen Gattungen: Dokufiktion 1/2 (2006), 142-154. 
gen, die eine (zusätzliche) Faktualitätsintention signalisieren - der Fiktionalitätspraxis entsprechend rezipieren sollen. ${ }^{35}$

In Ursula Krechels Landgericht gibt es nun aber eine Vielzahl von Sätzen, an denen sich zeigen lässt, dass genau dieses vom Kompositionalismus nahegelegte Vorgehen verfehlt wäre. So wird das integrierte dokumentarische Material etwa häufig kommentiert und bewertet, und zwar nicht in kursivierter Form - also scheinbar im fiktionalen Diskurs. Die folgenden Beispiele, die stets unmittelbar an kursivierte Passagen anschließen, stehen dabei exemplarisch für eine Fülle von weiteren Vorkommnissen:

Hatte das Justizministerium seinem [Kornitzers] Vorgesetzten, dem Landgerichtspräsidenten, seinen Antrag weitergereicht und um Stellungnahme gebeten? Vermutlich. ${ }^{36}$

Wie und bei wem ist dieser Eindruck entstanden? Bei den Parteien? Bei seinen Beisitzern? Haben sie den Vorsitzenden der Kammer angeschwärzt? ${ }^{37}$

Das war vornehm distanziert, aber in der Sache hart formuliert. ${ }^{38}$

Und besonders eindrücklich in der folgenden Passage:

(Auf dem erhaltenen Blatt seiner Erklärung befindet sich an dieser Stelle eine Bleistiftbemerkung eines, nein: d e s Leiters der nachfolgenden Untersuchung: Also Presse nicht wegen der Sache 1059/53? Das war in der Tat die Frage. War eine außerordentliche Aufmerksamkeit vom Verhandlungsführenden Richter initiiert worden? Oder war die Öffentlichkeit schon auf verschiedene Weise instruiert, und Landgerichtsdirektor Zeh mußte den Fall aus nächster Nähe dokumentieren? $)^{39}$

Wer denkt hier nach? Wer spekuliert und kommentiert hier? Da die zitierten Passagen nicht kursiviert sind, müsste man eigentlich annehmen, dass die Fiktionalitätskonvention wirksam ist - und dass es sich dabei also um Überlegungen der Erzählinstanz handelt. Aber ist diese Annahme gerechtfertigt? Ist fernerhin

35 Vgl. zu diesem in Anlehnung an das „Reality Principle“ formulierten „Fictionality Principle“ Konrad: Dimensionen der Fiktionalität, 466: „Alle Äußerungen in einem fiktionalen Text, bei denen Faktualitätssignale dies nicht ausdrücklich negieren, sind ausschließlich dem fiktionalen Diskurs zuzurechnen. Mit anderen Worten: Alle Äußerungen, die nicht durch textimmanente Signale anders gekennzeichnet sind, sind als rein fiktionalen [sic] Aussagen zu interpretieren." Vgl. dazu auch Gertken und Köppe, die völlig zu Recht behaupten, dass für „den Status eines Texts als fiktional [...] diese primäre Absicht [d.h. die Fiktionalitätsintention] und nur sie ausschlaggebend“ ist (Gertken/Köppe: „Fiktionalität“, 256, Fn. 80).

36 Krechel: Landgericht, 417.

37 Krechel: Landgericht, 466.

38 Krechel: Landgericht, 466.

39 Krechel: Landgericht, 430-431. 
davon auszugehen, dass diese Sätze von der Autorin überhaupt mit einer Fiktionalitätsintention verfasst wurden? Soll sich der Leser also vorstellen, dass eine Passage aus einem bestimmten Brief ,vornehm distanziert, aber in der Sache hart formuliert" war? Oder handelt es sich doch vielmehr um einen faktualen Diskurs, in dem die Autorin selbst über reale Sachverhalte spekuliert? Oder stimmt möglicherweise sogar beides?

In meinen Augen gibt es in Bezug auf diese und alle vergleichbaren Stellen nur eine ehrliche Antwort: Es lässt sich nicht mit Sicherheit sagen. Eine Entscheidung zugunsten der Fiktionalität und/oder der Faktualität ist hier nicht zweifelsfrei zu treffen, sodass die kompositionalistische Analyse ins Leere laufen muss. Denn gerade gegen diese für Dokufiktion spezifische Unsicherheit in Bezug auf den Textstatus hilft auch der Kompositionalismus nicht, ja, er geht in dieser Hinsicht sogar am Wesentlichen der Dokufiktion vorbei: Was mit Ursula Krechels Landgericht vorliegt, ist kein fiktionaler Text, in den nur einige dokumentarische bzw. faktuale Einschübe integriert wären, die den fiktionalen Text glaubwürdiger erscheinen lassen sollen. ${ }^{40}$ Das Dokumentarische hat nicht nur Anteil an der Fiktion und ergänzt diese, sondern umgekehrt ergänzt und belebt auch die Fiktion das Dokumentarische. ${ }^{41}$ Agnes Bidmon etwa weist auf die „Emotionalisierung des Faktischen mithilfe der Fiktion“ hin, wodurch „eine besonders nachhaltige Auseinandersetzung mit dem Text befördert“ “42 werde. Anstelle eines global fiktionalen Textes mit faktualen Einschüben ist also von einer für dokufiktionale Texte spezifischen Durchsetzung, einer permanenten Wechselwirkung von Fakt und

40 Dass Landgericht auf dem Titelblatt als „Roman“ bezeichnet wird, ist kein Gegenargument. Wie bereits erläutert, ist es für dokufiktionale Texte vielmehr gerade kennzeichnend, dass die Signale in Bezug auf die Intention des Autors in unterschiedliche Richtungen weisen, sich also Fiktionalitäts- neben Faktualitätssignalen finden. Dementsprechend wäre das Problem auch damit nicht zu lösen, dass der Gesamttext als faktual interpretiert würde, denn Ursula Krechels Landgericht ist ebenso wenig ein faktualer Text, in den nur einige fiktionale Einschübe integriert wären.

41 Vgl. dazu Ulrich Rüdenauer, der feststellt, es gelinge Krechel, „das Dokumentarische und Fiktionale im Roman auf kunstvolle Weise zu verknüpfen, zu verweben, sodass die Übergänge noch kenntlich sind, aber das Gefundene vom Erfundenen davon getragen [sic] wird und das Erfundene vom Gefundenen getragen.“ (Rüdenauer, Ulrich. „Die Archivarin des Verdrängten“. In: Zeit online vom 09.10.2012, https://www.zeit.de/kultur/literatur/2012-10/buchpreis-2012-ursulakrechel (24.03.2020)). In ähnlicher Weise spricht auch Friederike Gösweiner von einem „Ineinanderflechten von Fakt und Fiktion“ (Gösweiner: „Ein jüdischer Kohlhaas“).

42 Bidmon: „Streng vertraulich!“, 430 (beide Zitate). Vgl. zur Verschränkung von Wissensvermittlung und emotionaler Anteilnahme im (insgesamt etwas anders gelagerten) Dokudrama Steinle, Matthias. „Geschichte im Film: Zum Umgang mit den Zeichen der Vergangenheit im Dokudrama der Gegenwart“. In: History Goes Pop. Zur Repräsentation von Geschichte in populären Medien und Genres. Hg. Barbara Korte, Sylvia Paletschek. Bielefeld: transcript 2009, 147-165. 
Fiktion auszugehen. Die Verschränkung von „dokumentarische[n] und fiktionale[n] Elementen, Verfahren und Strategien“43, von der Christian von Tschilschke spricht, betrifft somit nicht etwa nur einzelne Äußerungen, sondern potenziell jeden einzelnen Satz des dokufiktionalen Textes. Sowohl hinsichtlich der Charakterisierung einzelner Textpassagen als auch in Bezug auf den Umgang mit dem Gesamttext ermöglicht der Kompositionalismus also letztlich kein angemessenes Urteil über Dokufiktionen. Auch wenn er fraglos geeigneter zur Analyse dieser Texte ist als seine unmittelbaren Konkurrenten, stößt er bei der Dokufiktion doch in mehrerlei Hinsicht an seine Grenzen - ein Scheitern, das meines Erachtens ganz bewusst von Dokufiktionen provoziert wird.

\section{3}

Ich möchte deshalb für die dritte Option im Umgang mit dokufiktionalen Texten plädieren: Meiner Auffassung nach ist Dokufiktion als ein echter sogenannter „Grenzfall“ zu verstehen, d.h. als Textform, bei der eine nicht aufzulösende Unsicherheit darüber besteht, ob die entsprechenden Werke mit einer Fiktionalitätsintention hervorgebracht wurden oder nicht (und welchen Konventionen dementsprechend auch bei der Rezeption zu folgen wäre). ${ }^{44}$ Aus einer fiktionalitätstheoretischen Perspektive wird der Dokufiktion die Analyse als Grenzfall am besten gerecht, weil sie die Irritation über die Gattungszugehörigkeit dieser Werke, wie sie schon aus der einleitend zitierten Rezension von Friederike Gösweiner sprach, ${ }^{45}$ angemessen widerspiegelt: In meinen Augen ist es tatsächlich fundamental unklar, ob dokufiktionale Texte als fiktionale oder als faktuale intendiert sind und wie sie deshalb zu behandeln sind. ${ }^{46}$ Diese Analyse als Grenz-

43 von Tschilschke: „Biographische Dokufiktion in der spanischen Literatur der Gegenwart“, 381.

44 Vgl. Köppe: Literatur und Erkenntnis, 45, sowie Gertken/Köppe: „Fiktionalität“, 258-259. Auch Klein und Martínez besprechen eine ganze Reihe von „Borderline-Texten“ bzw. „Grenzfälle[n]“ (Klein/Martínez: „Wirklichkeitserzählungen“, 4), ohne jedoch konkret auf Dokufiktionen Bezug zu nehmen. Wichtig ist zudem, dass Dokufiktionen nicht unter die von Klein und Martínez behandelten „Wirklichkeitserzählungen“ fallen, da diese laut Klein und Martínez - ganz anders als Dokufiktionen - „nicht literarisch in einem engeren Verständnis sind, weil sie [...] keinen hohen Grad an Poetizität aufweisen“ (Klein/Martínez: „Wirklichkeitserzählungen“, 6).

45 Vgl. Gösweiner: „Ein jüdischer Kohlhaas“. Vgl. ähnlich auch Andreas Isenschmid, der Landgericht als „Doppelwesen aus historischer Recherche und Roman“ bezeichnet (Isenschmid, Andreas. „Ursula Krechel. Fremd im eigenen Land“. In: Die Zeit vom 11.10.2012, 51).

46 Wohlgemerkt impliziert die Akzeptanz von Grenzfällen nicht die Einführung eines dritten Bereiches zwischen fiktionalem und faktualem Diskurs, wie ihn z. B. Marie-Laure Ryan mit den 
fall scheint mir auch der Interpretation von Agnes Bidmon zu entsprechen, die dokufiktionale Texte explizit als „Grenzgänger-Texte“47 bezeichnet und deshalb dafür plädiert, den Umgang mit ihnen auf einen „semidokumentarischen Pakt“48 zu gründen, der darin besteht, „dass die Texte auf vielfältige Weise entweder andeuten oder offen anzeigen, dass sie zugleich referentiell und konstruktiv verfahren." 49 Auch dieser Vorschlag trägt der Sonderstellung der Dokufiktion zwischen Fiktionalität und Faktualität Rechnung, zieht aus der Einordnung als Grenzfall jedoch eine andere Konsequenz, als ich sie abschließend vorschlagen möchte.

Wie Jan Gertken und Tilmann Köppe darlegen, gibt es grundsätzlich drei (sich wechselseitig nicht ausschließende) Möglichkeiten des Umgangs mit Grenzfällen:50 Entweder man nimmt an, dass Grenzfälle sowohl fiktional als auch faktual sind, dass die Unterscheidung zwischen Fiktionalität und Faktualität also nicht exklusiv ist. Oder man nimmt an, dass Grenzfälle weder fiktional noch faktual sind, dass die Unterscheidung zwischen Fiktionalität und Faktualität also nicht vollständig ist. Oder man nimmt an, dass der Status von Grenzfällen unauflösbar vage bleibt, d. h. dass unentscheidbar ist, ob sie fiktional, faktual, beides oder keines von beidem sind. Agnes Bidmon plädiert mit der Aussage, dass dokufiktionale Texte ,zugleich referentiell und konstruktiv verfahren“51, für die nicht-Exklusivität der Unterscheidung. Der Vorschlag eines „semidokumen-

„three classes: fiction, nonfiction, and ,inbetween“ “ annimmt (Ryan, Marie-Laure. Avatars of Story. Minneapolis/London: University of Minnesota Press 2006, 54). Grenzfälle sind kein Drittes zwischen fiktionalem und faktualem Diskurs, sondern Fälle, in denen keine Klarheit darüber besteht, welchem der beiden Diskurse ein Text zugehört. Zugestanden wird damit also lediglich das Vorhandensein von Texten, die sich nicht eindeutig als fiktional oder faktual klassifizieren lassen.

47 Bidmon: „Streng vertraulich!“, 430. Bidmon stellt darüber hinaus fest, dokufiktionales Erzählen sei „bewusst als Grenzgang zwischen Fakt und Fiktion angelegt“ (Bidmon: „Streng vertraulich!“, 423). Auch Antonius Weixler bezeichnet „Dokufiction“ als eine „innovative[] Hybridgattung[]“ (Weixler, Antonius. „Authentisches erzählen - authentisches Erzählen. Über Authentizität als Zuschreibungsphänomen und Pakt“. In: Authentisches Erzählen. Produktion, Narration, Rezeption. Hg. Antonius Weixler. Berlin/Boston: De Gruyter 2012, 1-32, hier 9).

48 Bidmon: „Streng vertraulich!“, 430.

49 Bidmon: „Streng vertraulich!“, 430.

50 Vgl. Gertken/Köppe: „Fiktionalität“, 259.

51 Bidmon: „Streng vertraulich!“, 430. Vgl. auch Philipp Hubmann, der in Bezug auf Joachim Gaertners Ich bin voller Hass - und das liebe ich!! - ein Text, den Antonius Weixler einleitend als „dokumentarische[n] Roman“ (Weixler: „Authentisches erzählen - authentisches Erzählen“, 25) bezeichnet - zusammenfassend festhält: „Durch diese Verortung des Romans zwischen Dokumentation und Poesie wird augenscheinlich auch darauf verzichtet, einen bestimmten Kommunikationskanal, sei es der faktuale oder fiktionale, exklusiv zu nutzen“ (Hubmann, Philipp. „Dokumente des Amoks. Literarische Montage als narrative Authentifizierungsstrategie am Beispiel von Joachim Gaertners Roman Ich bin voller Hass - und das liebe ich!!“. In: Authentisches 
tarischen Pakt[es]“"52 scheint darüber hinaus auch für die Unvollständigkeit der Unterscheidung zu sprechen, wird damit doch eine dritte Kategorie zwischen Fiktionalität und Faktualität angenommen, für die offenbar eigene Konventionen gelten sollen. Auch all dies lässt sich unproblematisch in den Rahmen einer institutionellen Fiktionalitätstheorie einfügen: Gerade institutionelle Theorien sind mit der Idee vereinbar, dass für bestimmte Gattungen, Genres oder Darstellungsweisen je spezifische Praktiken der Produktion und Rezeption existieren.

Dass ich hier dennoch für die dritte Variante - und damit für die unauflösliche Vagheit - plädieren möchte, liegt nicht nur daran, dass sich (wie zuvor gezeigt) in Bezug auf viele Passagen in dokufiktionalen Texten nicht sicher entscheiden lässt, ob sie als fiktionaler und/oder faktualer Diskurs intendiert (und deshalb auch in dieser Weise zu behandeln) sind, sondern vor allem daran, dass mir die provokative Zwischenstellung dokufiktionaler Texte zwischen Fiktionalität und Faktualität als eine bewusst gewählte erscheint. ${ }^{53}$ Nimmt man für dokufiktionale Texte eine eigene Sonderkategorie an, wie es ein „semidokumentarischer Pakt“ suggeriert, wäre diese bewusste Provokation, dieser bewusste Grenzlauf zwischen den Kategorien theoretisch eingehegt und den Regeln eines konventionalisierten Umgangs - in diesem Fall also denjenigen des „semidokumentarischen Paktes“ unterworfen. Meines Erachtens läuft dies der Intention, die aus der speziellen Konstruktion und Darstellungsweise dokufiktionaler Texte spricht, jedoch gerade entgegen: Sobald die Provokation zur Konvention wird, wird sie unwirksam.

\section{Literatur}

Bidmon, Agnes. „Streng vertraulich! Dokufiktionales Erzählen als Schreibweise des Politischen in der Literatur der Gegenwart anhand von Ilija Trojanows Macht und Widerstand“. In: Politische Literatur. Begriffe, Debatten, Aktualität. Hg. Christine Lubkoll, Manuel Illi, Anna Hampel. Stuttgart: J.B. Metzler 2019, 421-440.

Erzählen. Produktion, Narration, Rezeption. Hg. Antonius Weixler. Berlin/Boston: De Gruyter 2012, 145-175, hier 171-172).

52 Bidmon: „Streng vertraulich!“, 430.

53 Trotzdem ist der unterschiedliche Umgang mit Dokufiktionen als Grenzfall sicher auch dem konkreten dokufiktionalen Text geschuldet, der im Fokus der Untersuchung steht. Insofern lässt es sich wohl nur von dokufiktionalem Grenzfall zu dokufiktionalem Grenzfall endgültig entscheiden, ob der Text am besten als fiktional und faktual, weder fiktional noch faktual und/oder in dieser Hinsicht unbestimmt beschrieben werden sollte. Mein Urteil bezieht sich auf die Textsorte als solche, schließt damit aber natürlich nicht aus, dass bestimmte dokufiktionale Texte möglicherweise einen anderen Schwerpunkt legen. 
Blume, Peter. Fiktion und Weltwissen. Der Beitrag nichtfiktionaler Konzepte zur Sinnkonstitution fiktionaler Erzählliteratur. Berlin: Erich Schmidt 2004.

Gertken, Jan/Köppe, Tilmann. „Fiktionalität“. In: Grenzen der Literatur. Zu Begriff und Phänomen des Literarischen. Hg. Simone Winko, Fotis Jannidis, Gerhard Lauer. Berlin/New York: De Gruyter 2009, 228-266.

Gösweiner, Friederike. „Ein jüdischer Kohlhaas. Ursula Krechels großer Roman ,Landgericht' über das Schicksal eines jüdischen Exilanten, der nach Deutschland zurückkehrt und vergeblich nach Gerechtigkeit sucht“. In: literaturkritik.de vom 08.10.2012. https://literaturkritik.de/public/rezension.php (24.03.2020).

Hahnemann, Andy. ,,Footnotes are real'. Populäre Literatur als Medium der Wissensvermittlung“. In: Non Fiktion. Arsenal der anderen Gattungen: Dokufiktion 1/2 (2006), 142-154.

Hammelehle, Sebastian. „Deutsche-Buchpreisträgerin Ursula Krechel: Die Gerechtwerderin“. In: Spiegel online vom 09.10.2012. https://www.spiegel.de/kultur/literatur/ danksagung-der-buchpreistraegerin-ursula-krechel-in-frankfurt-am-main-a-860202.html (24.03.2020).

Hubmann, Philipp. „Dokumente des Amoks. Literarische Montage als narrative Authentifizierungsstrategie am Beispiel von Joachim Gaertners Roman Ich bin voller Hass - und das liebe ich!!“. In: Authentisches Erzählen. Produktion, Narration, Rezeption. Hg. Antonius Weixler. Berlin/Boston: De Gruyter 2012, 145-175.

Huck, Christian. „Authentizität im Dokumentarfilm. Das Prinzip des falschen Umkehrschlusses als Erzählstrategie zur Beglaubigung massenmedialen Wissens“. In: Authentisches Erzählen. Produktion, Narration, Rezeption. Hg. Antonius Weixler. Berlin/Boston: De Gruyter 2012, 239-264.

Isenschmid, Andreas. „Ursula Krechel. Fremd im eigenen Land“. In: Die Zeit vom 11.10.2012, 51.

Klein, Christian/Martínez, Matías. „Wirklichkeitserzählungen. Felder, Formen und Funktionen nicht-literarischen Erzählens“. In: Wirklichkeitserzählungen. Felder, Formen und Funktionen nicht-literarischen Erzählens. Hg. Christian Klein, Matías Martínez. Stuttgart/Weimar: J.B. Metzler 2009, 1-13.

Konrad, Eva-Maria. Dimensionen der Fiktionalität. Analyse eines Grundbegriffs der Literaturwissenschaft. Münster: mentis 2014.

Köppe, Tilmann. „Die Institution Fiktionalität“. In: Fiktionalität. Ein interdisziplinäres Handbuch. Hg. Tobias Klauk, Tilmann Köppe. Berlin/Boston: De Gruyter 2014, 35-49.

Köppe, Tilmann. Literatur und Erkenntnis. Studien zur kognitiven Signifikanz fiktionaler literarischer Werke. Paderborn: mentis 2008.

Krechel, Ursula. Landgericht. München: btb 2014 [2012].

Lamarque, Peter/Olsen, Stein Haugom. Truth, Fiction, and Literature. A Philosophical Perspective. Oxford: Oxford University Press 2002 [1994].

Martínez, Matías/Scheffel, Michael. Einführung in die Erzähltheorie. München: C.H.Beck 2002 [1999].

Möller, Barbara. „Der verzweifelte Kampf des Richard Kornitzer“. In: Die Welt vom 30.01.2017. https://www.welt.de/kultur/medien/article161634391/Der-verzweifelte-Kampf-desRichard-Kornitzer.html (07.10.2020).

Oels, David/Porombka, Stephan/Schütz, Erhard. „DokuFiktion - Editorial“. In: Non Fiktion. Arsenal der anderen Gattungen: Dokufiktion 1/2 (2006), 108-111. 
Platthaus, Andreas: „Ursula Krechels ,Landgericht‘. In der Sache Kornitzer“. In: Frankfurter Allgemeine Zeitung vom 10.10.2012. https://www.faz.net/aktuell/feuilleton/buecher/ rezensionen/belletristik/ursula-krechels-landgericht-in-der-sache-kornitzer-11912092. html?printPagedArticle=true\#pagelndex_2 (07.10.2020).

Picaper, Ilse. „(Re)Lectures. Sie wollte Zeugin sein. Zu Ursula Krechel, Landgericht. Roman“. In: Germanica 59 (2016), 205-212.

Rüdenauer, Ulrich. „Die Archivarin des Verdrängten“. In: Zeit online vom 09.10.2012 https: //www.zeit.de/kultur/literatur/2012-10/buchpreis-2012-ursula-krechel (24.03.2020).

Ryan, Marie-Laure. Avatars of Story. Minneapolis/London: University of Minnesota Press 2006.

Searle, John R. „The Logical Status of Fictional Discourse“ [1974/75]. In: Expression and Meaning. Studies in the Theory of Speech Acts. Hg. John R. Searle. Cambridge: Cambridge University Press 1979, 58-75.

Steinle, Matthias. „Geschichte im Film: Zum Umgang mit den Zeichen der Vergangenheit im Dokudrama der Gegenwart“. In: History Goes Pop. Zur Repräsentation von Geschichte in populären Medien und Genres. Hg. Barbara Korte, Sylvia Paletschek. Bielefeld: transcript 2009, 147-165.

Tolstoi, Lew. Anna Karenina. Aus dem Russischen neu übersetzt und kommentiert von Rosemarie Tietze. München: Carl Hanser 2013 [1878].

Tschilschke, Christian von. „Biographische Dokufiktion in der spanischen Literatur der Gegenwart. Las esquines del aire von Juan Manuel de Prada und Soldados de Salamina von Javier Cercas“. In: Literatur als Lebensgeschichte. Biographisches Erzählen von der Moderne bis zur Gegenwart. Hg. Peter Braun, Bernd Stiegler. Bielefeld: transcript 2012, 377-400.

Weber, Hermann. „Landgericht. Roman. Von Ursula Krechel“. In: Neue juristische Wochenschrift 66.11 (2013), 762-763.

Weixler, Antonius. „Authentisches erzählen - authentisches Erzählen. Über Authentizität als Zuschreibungsphänomen und Pakt“. In: Authentisches Erzählen. Produktion, Narration, Rezeption. Hg. Antonius Weixler. Berlin/Boston: De Gruyter 2012, 1-32.

White, Hayden. Metahistory. The Historical Imagination in Nineteenth-Century Europe. Baltimore/London: Johns Hopkins University Press 1973.

Wiegandt, Markus. Chronisten der Zwischenwelten. Dokufiktion als Genre. Operationalisierung eines medienwissenschaftlichen Begriffs für die Literaturwissenschaft. Heidelberg: Winter 2017. 
